\title{
Investigation of Nucleation and Growth of Detwinning Mechanism in Martensitic Single Crystal NiTi Using Digital Image Correlation
}

\author{
Savas Dilibal
}

Received: 15 May 2013/Revised: 19 June 2013/Accepted: 23 June 2013/Published online: 23 July 2013

(C) Springer Science+Business Media New York and ASM International 2013

\begin{abstract}
In this study, the deformation of martensitic NiTi was investigated utilizing single crystal Ti-50.1 at.\% $\mathrm{Ni}$ in [123], [111], and [001] orientations. The stress-strain response of the martensitic NiTi was resolved experimentally using incremental tensile loading. The nucleation and growth of the detwinning mechanism was investigated by means of in-situ digital image correlation (DIC) technique which enabled the precise determination of the onset of detwinning on the stress-strain curve. The mechanism of detwinning nucleation was observed before the stress plateau on the stress-strain curve. The use of DIC for the [123] orientation revealed that the intersection of twins causes more hardening through twinning-induced strains. Based on the stress-strain response of the [123], [111], and [001] orientations, it is obvious that detwinning is orientation independent. In contrast, the evolution of detwinning after nucleation is orientation dependent.
\end{abstract}

Keywords Shape memory alloys $\cdot \mathrm{NiTi} \cdot$ Martensite Twinning $\cdot$ Detwinning $\cdot$ Digital image correlation

\section{Introduction}

Shape memory alloys contribute to applications ranging from aerospace and biomedical fields to electronics and automotive industries, with their superior shape memory effect and pseudoelastic properties [1]. The NiTi alloy of near equiatomic composition undergoes a martensitic phase transformation from cubic lattice (B2) austenite to

S. Dilibal $(\bowtie)$

Department of Biomedical Engineering, Bahcesehir University, Ciragan Caddesi, Besiktas, 34353 Istanbul, Turkey

e-mail: savas.dilibal@bahcesehir.edu.tr monoclinic $\left(\mathrm{B} 19^{\prime}\right)$ martensite structure upon cooling or upon straining. Once the NiTi (B2 lattice) alloy is cooled in a stress-free environment, the monoclinic $\left(\mathrm{B} 19^{\prime}\right) \mathrm{NiTi}$ selfaccommodated martensite variants form. Many selfaccommodating combinations of martensite variants initially form while conserving the alloy's macroscopic shape [2]. During deformation in the martensitic phase, preferentially oriented martensitic variants undergo detwinning and mechanical twinning subsequently. Detwinning is the transition from multiple variants to the formation of a single variant. Upon heating, the shape memory effect occurs by reverse transformation. Pseudoelasticity is the other important characteristic of shape memory alloys [3]. The complete pseudoelastic behavior takes place when the alloy is stressed between the austenite final $\left(A_{\mathrm{f}}\right)$ and $M_{\mathrm{d}}$ temperatures, where $M_{\mathrm{d}}$ is the temperature above which martensite cannot be stress induced. The recovery of deformation occurs upon unloading $[4,5]$. While the selfaccommodated martensite variants form in thermally induced martensite without applied load, favorably oriented martensite variants form in stress induced martensite under applied load.

In the deformed NiTi martensite, the existence or coexistence of different types of twins was discovered experimentally via transmission electron microscopic (TEM) and high resolution transmission electron microscopic (HRTEM) observations in previous studies [6-14]. Among the twin modes, Type II- 1 twin was the predominant transformation twinning mode that was found experimentally in solutionized NiTi [6]. Through HRTEM observations on polycrystalline NiTi martensite [7] and HRTEM image simulation [8], Xie and Liu have proved that the $\langle 011\rangle$ type II twin is atomically rational. The detwinning process is the growth of one variant at the expense of other. It is well known that this process is a 
unique deformation mechanism which plays a significant role in the shape memory effect. Detwinning of the Type II-1 transformation twins starts at the early stages of deformation. Various additional twin modes were observed on the deformation twinning mechanism. The deformation twin of the (001) type mainly occurs at moderate strain levels. Ezaz et al. [9] observed the (001) twin modes at lower stresses and (100) twin modes at higher stresses in their TEM analysis. Nishida et al. [10] discovered (201) twin modes in martensite twins during deformation experiments. With the appearance of secondary (201) twins and the existing (001) and (201)twins, the martensite microstructure becomes more complicated. More recently, Ezaz et al. [9, 11, 12] demonstrated the deformation twinning process using energy barriers via atomistic simulations. They have also calculated the energy barriers and noted that twinning is favored over slip during the deformation mechanism of NiTi in martensite phase [12].

Many studies have been conducted for a complete understanding of the detwinning mechanism of NiTi martensite [6-12]. In this study, the nucleation and growth of the detwinning mechanism was investigated by measuring the local strain evolution and the characteristic strains associated with the martensitic phase. In-situ experiments which were performed using an advanced digital image correlation (DIC) technique provided a unique opportunity to monitor and analyze full-field and local strain evolution during tensile loading.

\section{Experimental Procedure}

Ti-50.1 at.\% $\mathrm{Ni}$ single crystal samples oriented in the [123], [111], and [001] directions were used in this work. The samples were solutionized at $1000{ }^{\circ} \mathrm{C}$ for $2 \mathrm{~h}$ in an inert gas atmosphere. Following the solutionizing process, they were heat treated at $450{ }^{\circ} \mathrm{C}$ for $100 \mathrm{~h}$ and water quenched. As a result of the heat treatment, a fully martensitic structure was obtained at room temperature. Differential scanning calorimetry (DSC) results were an austenite start temperature of $45{ }^{\circ} \mathrm{C}$ and a martensite start of $40{ }^{\circ} \mathrm{C}$ (see Fig. 1a).

Tensile tests were conducted on small dog bone specimens (width $2.73 \mathrm{~mm}$, length $26.63 \mathrm{~mm}$, and thickness $1.21 \mathrm{~mm}$ ) in the martensitic phase at room temperature $\left(28{ }^{\circ} \mathrm{C}\right)$. Full-field strains (macroscopic strain) were measured with a miniature extensometer (5 $\mathrm{mm}$ gage length) to produce the full-field stress-strain curves.

DIC was used to investigate the evolution of detwinning during martensitic phase deformation. This technique gives further insight into full-field and local strain measurements via in-situ or ex-situ observation. DIC has been utilized in studies ranging from the pseudoelastic response of NiMnGa [15] and the phase transformation volume change in CoNiAl [16] to the effect of grain size on local deformation [17], fatigue crack behavior [18], and full-field strain evolution in $\mathrm{NiFeGa}$ [19]. It is also used on polycrystalline NiTi to obtain the full-field data under mechanical cycling [20] and shear dominant loading [21]. The DIC technique measures displacement fields by tracking features on the specimen surface with a random speckle pattern. To perform DIC, appropriately sized stable speckles were applied to the surface of the polished specimen using an airbrush (see Fig. 1b). Upon tensile loading, images of the specimen surface were captured with a CCD camera $(1600 \times 1200$ pixels) and an optical lens at $\times 5$ magnification for in-situ observation. Images were captured using mechanical testing software which also controlled the servo-hydraulic load frame. The first captured image is chosen as the reference image. After selecting the area of interest (AOI) in the reference image, the AOI is divided into small square areas called subsets. The dimensions of the AOI were
Fig. 1 (a) DSC results for the Ti-50.1 at.\% Ni which was heat treated at $450{ }^{\circ} \mathrm{C}$ for $100 \mathrm{~h}$ then water quenched. (b) Speckle patterned specimen surface with AOI (the miniature extensometer legs are seen on both sides of the specimen) (a)

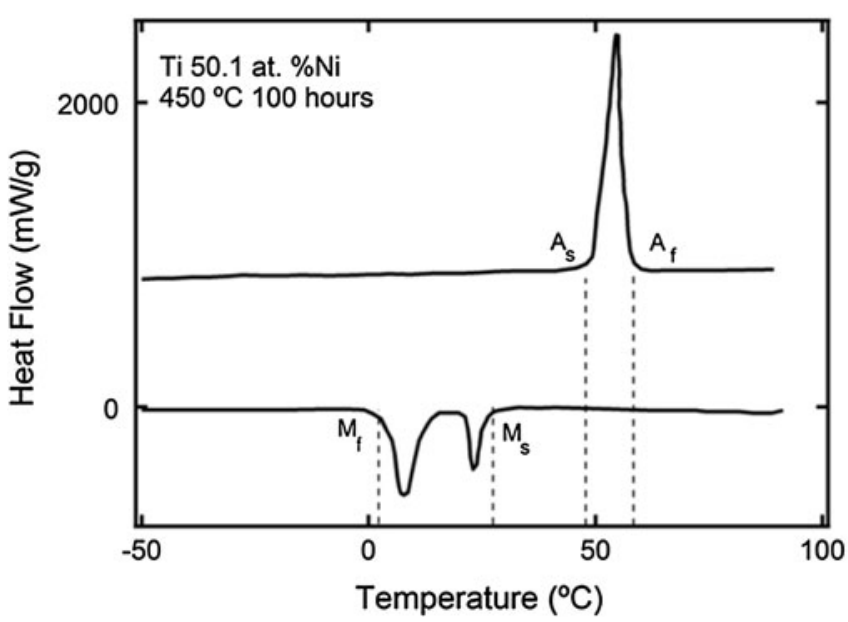

(b)

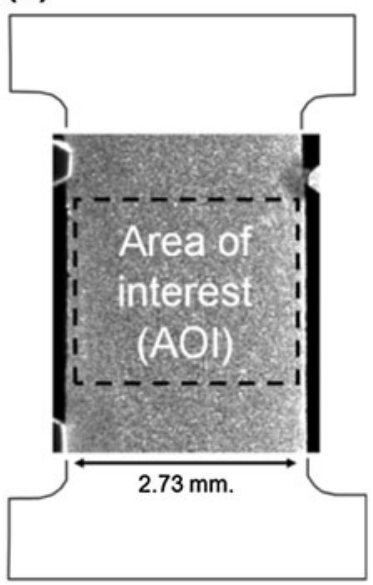


$2.5 \times 2.4 \mathrm{~mm}^{2}$ as seen in Fig. 1b. With DIC, all of the following images are compared against the reference image. DIC was performed using software developed by Correlated Solutions Inc. A subset size of $51 \times 51$ pixels and a subset spacing of 12 pixels were chosen to correlate the images. A contour map of strains was obtained by differentiating the DIC displacement fields. A general background on DIC and further details on its applications can be found in [22, 23]. In-situ mesoscopic observations were used to investigate the local strain evolution on detwinning bands.

The tensile stress-strain response of the NiTi single crystal is schematized into four stages, which are indicated in Fig. 2. In stage I, the self-accommodated martensite is internally twinned, with a predominant Type II- 1 transformation twin and two favorably oriented variants forming the correspondent-variant pair (CVP). In stage II, detwinning of the Type II-1 twin occurs following the CVP formation. In stage III, an upward gradient takes place on the stress-strain curve with the appearance of compound twins. In stage IV, the plastic flow of the mechanically twinned martensite occurs. In the schematic, a bold dashed line with an arrow is shown indicating the martensite to austenite transformation (reverse transformation) that occurs upon heating the specimen.

The specimens, oriented in the [123], [001], and [111] directions, underwent tensile tests consisting of $2 \%$ strain incremental loading until fracture at room temperature when they were in martensite phase. During the test process, at the end of each $2 \%$ strain increment, the tensile strain was unloaded. The combined incremental tensile tests results are superimposed in the main stress-strain diagram shown in Fig. 3. The specimen oriented in the [123] direction exhibited the steepest slope of stress-strain curve among the three orientations, followed by the [111] and [001] directions.

\section{Results and Discussion}

The results presented in this paper elucidated the local strain evolution of detwinning and the subsequent deformation process in single crystal NiTi martensite, based on DIC strain measurements.

The first incremental test result for the specimen oriented in the [123] direction with meso-scale DIC analysis is shown in Fig. 4. The self-accommodated stress-free microstructure was the reference image for the DIC analysis, which can be seen in Fig. 4a as a homogenous pink with zero strain. At the early stage of the first $2 \%$ tensile strain increment, the full-field strain became heterogeneous starting in Fig. 4b-c. The subsequent image shows a pink to purple transition, indicating $0.4 \%$ homogenous strain as seen in Fig. 4d. After this point, the light blue parallel bands appeared at $85 \mathrm{MPa}$. The detected local strain alteration (see Fig. 4e) indicates the nucleation of a light blue band with approximately $1.5 \%$ strain. This clearly reveals the initial appearance of the detwinning mechanism in the specimen. The growth of detwin bands through the specimen can be seen in further images (see Fig. 4f-i). The last image during the loading, Fig. $4 \mathrm{i}$, shows that the red parallel bands became the dominant mechanism in the system indicating a region of intense detwinning. Note that the maximum strain on the growing red bands is higher than $4 \%$, though the full-field strain is only $2 \%$. To examine the local strain evolution seen in the red bands, two different two-point DIC displacement gages (digital extensometer) were located on one of the detwin bands as
Fig. 2 Schematic for the stress-strain response of NiTi martensite. The strain-stress curve exhibits four stages based on the evolution of twinning modes

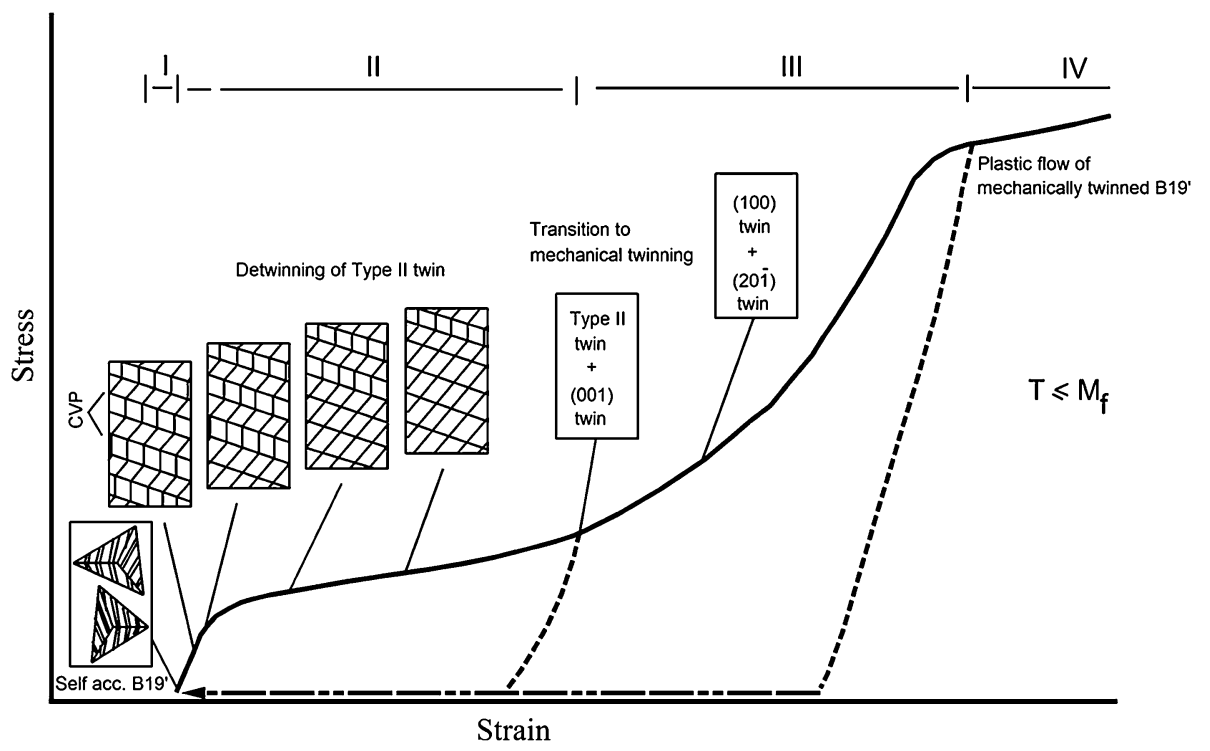


Fig. 3 The stress-strain response of the martensitic $\mathrm{NiTi}$ in [123], [111], and [001] orientations. The stress-strain curves for each orientation superimposed in the main graph

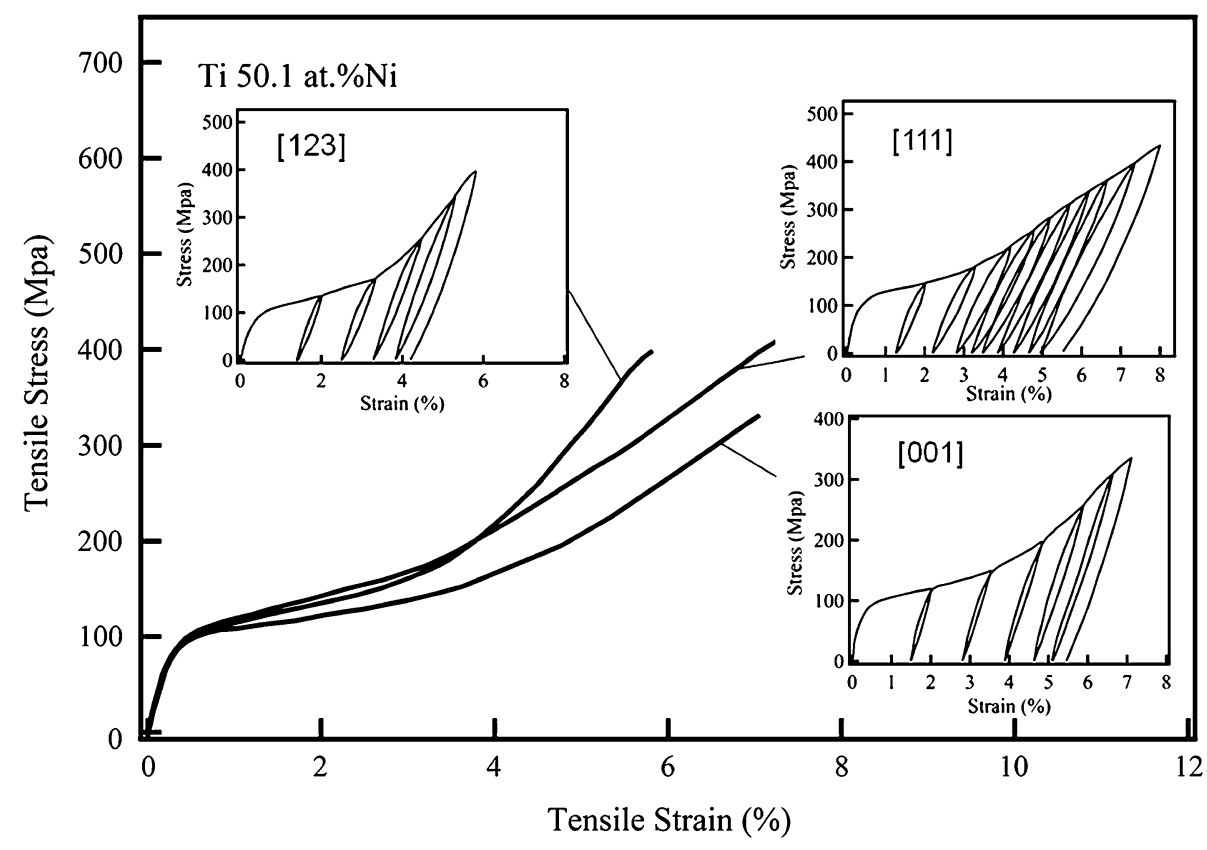

Fig. 4 The full-field and local (on the detwin band) stressstrain responses of NiTi in [123] orientation. Local strain measurement obtained from digital extensometers with two different lengths (images $i 1, i 2$ )

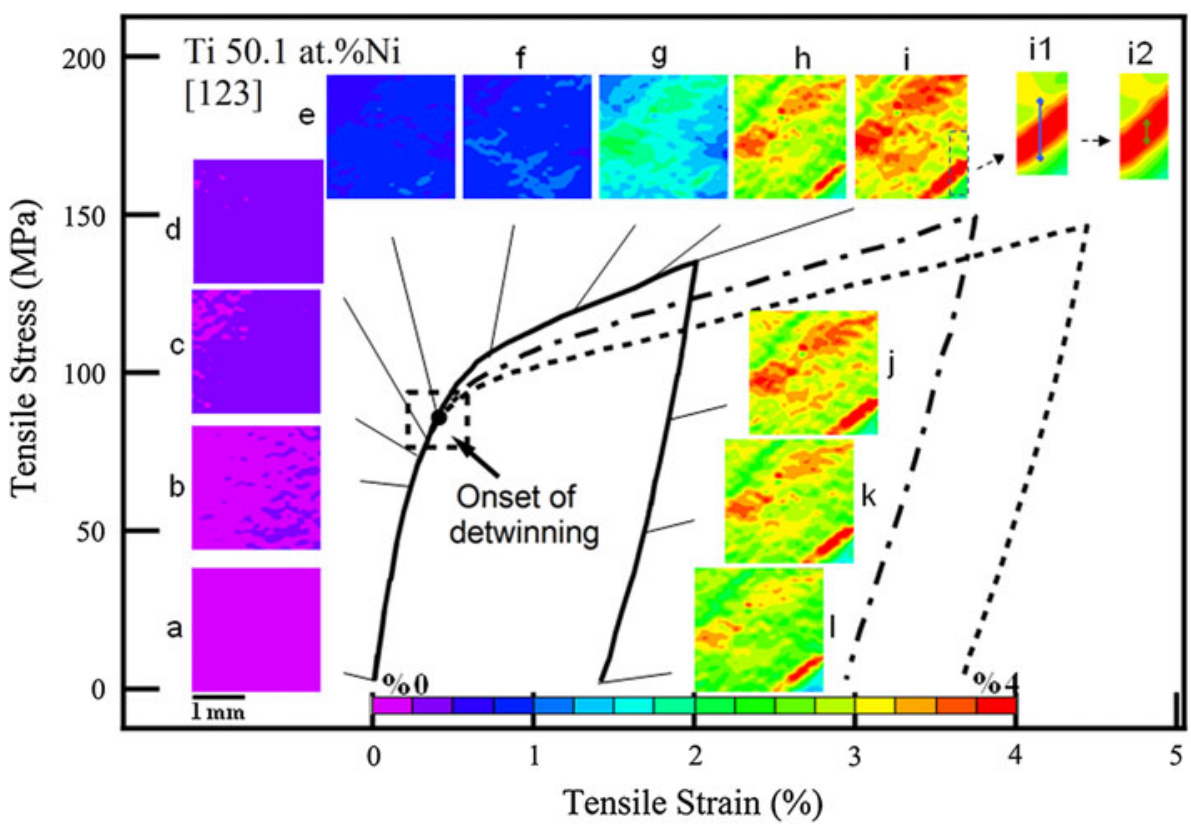

seen in Fig 4i1, i2. The stress-strain curves obtained from the two digital extensometer results are plotted with dashed lines on top of the miniature extensometer macroscopic stress-strain curve. The onset of detwinning of all three curves was found at $85 \mathrm{MPa}$. Upon unloading, a partial disappearance of the detwinned structure, determined via DIC, was observed, as shown in the last three images $(\mathrm{j}, \mathrm{k}$, and 1) in Fig. 4. This clarifies the reverse detwinning mechanism during unloading process.

In addition, the second incremental test and associated DIC results are seen in Fig. 5. The first image of the first
$2 \%$ strain increment was maintained as the re-reference image to perform DIC. At point $\mathrm{K}$, which is the intersection of the two curves $(\sim 130 \mathrm{MPa})$ in Fig. 5 , the slope of the second curve decreased until the $3.2 \%$ nominal strain. The detwinned bands became wider with the increase in load, and they covered the whole image. This confirmed that the detwinning process is almost completed in the second incremental test making a $40^{\circ}$ angle with the tensile direction. Upon unloading, the $\langle 011\rangle$ type II detwins partially revert back to twin as shown in Fig. $5 \mathrm{~h}-\mathrm{j}$. As observed from the second incremental test, the only 
Fig. 5 The first two incremental stress-strain response of the martensitic NiTi in [123] orientation

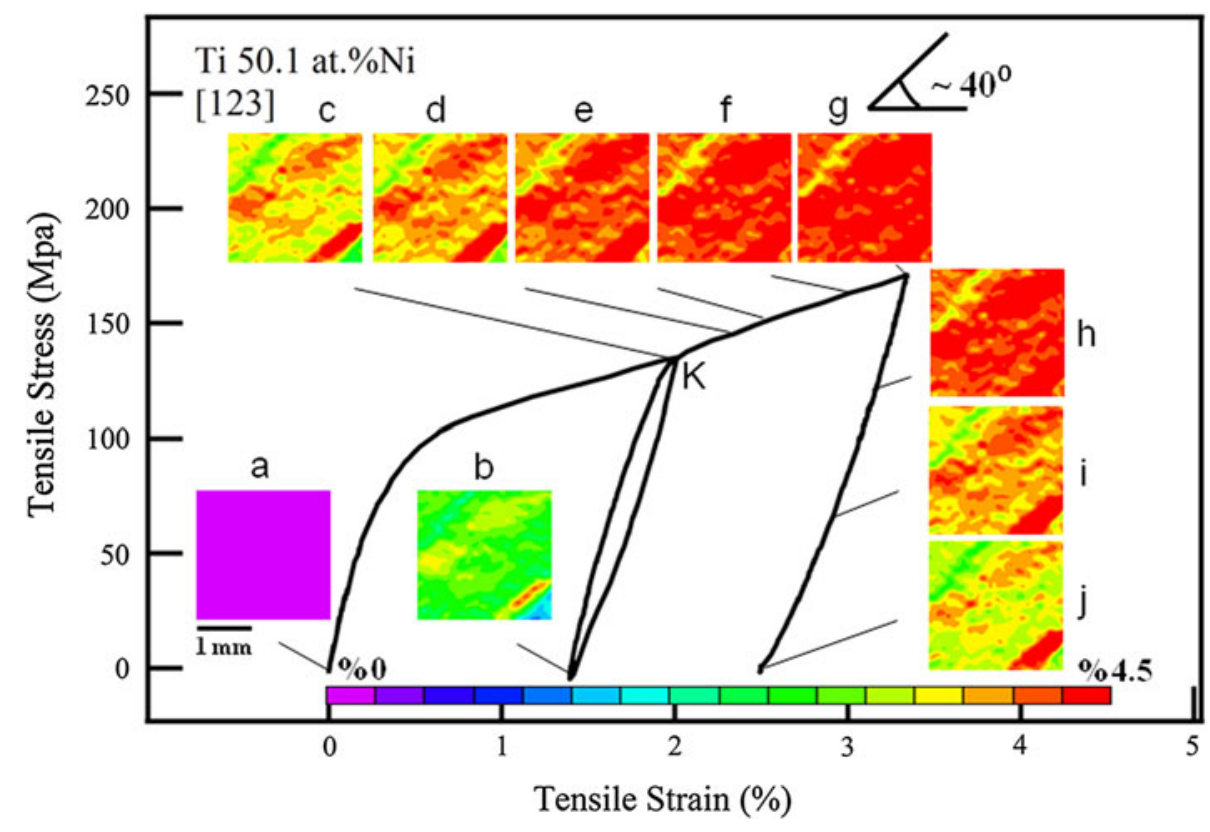

Fig. 6 The five incremental tests combined results for the martensitic NiTi in [123] direction. The meso-scale DIC result obtained from the end of the fifth incremental test. Dotted lines on the meso-scale DIC image show the occurrence of different twin modes

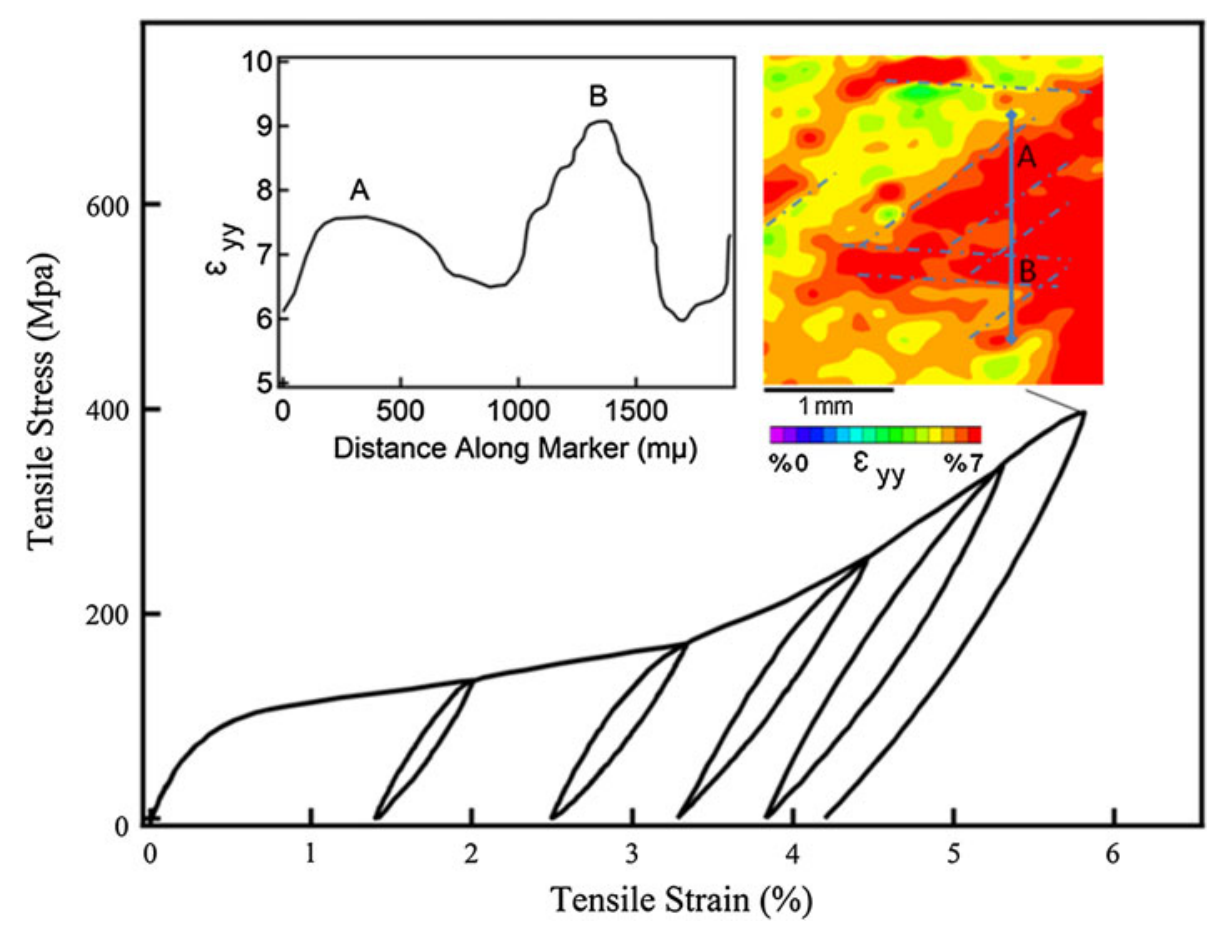

mechanism remaining in the microstructure is the detwinning mechanism which underwent up to $3.2 \%$ strain.

The five incremental tests combined results for the [123] case is illustrated in Fig. 6. After the detailed examination of the DIC result in Fig. 6, which was obtained at the end of the fifth incremental test before unloading, the nucleation of different twin modes was noticed as seen in Fig. 6. To measure the local strain alteration on different twin bands, a digital line marker was placed in the AOI using DIC software. The measured local strain along the marker was depicted as a diagram in Fig. 6. The detected highest strain was $9 \%$ and the lowest was $6 \%$ at the stress of $400 \mathrm{MPa}$. The dramatic fluctuation of the strain along the digital line marker shows the coexistence and intersection of different twin modes at the fifth incremental test for the [123] case. During the sixth incremental tensile test, the 
specimen was fractured upon loading. Fracture of the tensile loading precluded the study of plastic deformation up to large strains.

The local strain identified within detwin bands is higher than the applied strain on the specimen during deformation process. The rearrangement of the self-accommodated microstructure under the externally applied stress occurs up to a complete homogenous CVP formation according to full-field DIC analysis. Note that the onset of detwinning takes place after a homogenous microstructure formation.

The DIC technique not only shed light on the nucleation and propagation of the detwinning mechanism but also provides an insight into the occurrence of the reverse detwinning mechanism. The partial reverse detwinning and the gradual decline of the local strain on the parallel detwin bands was observed during unloading of each incremental test.

Liu et al. $[6,7]$ noted a stress-drop on the stress-strain curve during the transition of the detwinning mechanism for polycrystalline NiTi. In the single crystal case, a stressdrop was not observed on the stress-strain curve at the onset of detwinning. Furthermore, instead of a flat stress plateau at the second stage, an upward gradient was observed on the stress-strain curve up to $4 \%$ strain. The experimental results also demonstrated that the post detwinning deformation shows a steeper upward curvature at approximately $3.2 \%$ strain (see Fig. 3).

The stress-strain response of the martensitic NiTi in [123], [111], and [001] orientations was superimposed in a stress-strain diagram for better analysis of the differences. Among the three orientations, the [123] case exhibits a steep upward curvature in the stress-strain response at high strains. DIC results show that different twin interactions occur during the post detwinning process. Ezaz et al. [12] noted that the fundamental reason for the upward curvature is the twin interactions. They confirmed that (001) and (100) deformation twins interact with activated (201) twin modes and generate hardening of the martensitic NiTi. It is difficult to analyze clearly the twinning evolution due to the complicated interactions of the deformation mechanism and complex microstructural evolution during mechanical twinning at the stage III.

\section{Conclusions}

The detwinning process of B19' NiTi martensite has been investigated using DIC technique. The results are summarized as follows:

(1) The detwinning starts before the stress plateau during tensile loading. More than $4 \%$ local strain was measured using DIC on the detwin bands, while less than $2 \%$ full-field strain was applied.
(2) The DIC results pinpoint the precise stress level corresponding to the onset of detwinning. The onset of martensite detwinning stress is $85 \mathrm{MPa}$ for the [123] orientation.

(3) The DIC results of the [123] orientation show that twins intersect generates more hardening of the NiTi martensite with twinning-induced strains.

(4) The comparison of the stress-strain response of the [123], [001], and [111] shows that the nucleation stress for detwinning is orientation independent and the evolution of detwinning after nucleation is orientation dependent. The [123] orientation produces the steepest slope on the stress-strain curve at stage III compared to the [111] and [001] orientations.

Acknowledgments The author gratefully acknowledges Prof. Huseyin Sehitoglu (professor at the University of Illinois at Urbana-Champaign) for his support.

\section{References}

1. K. Otsuka, C.M. Wayman, Shape Memory Materials (Cambridge University Press, Cambridge, 1998)

2. S. Miyazaki, K. Otsuka, C.M. Wayman, The shape memory mechanism associated with the martensitic transformation in Ti-Ni alloys I. Self-accommodation. Acta Metall. 37, 1873-1884 (1989)

3. S. Miyazaki, K. Otsuka, Y. Suzuki, Transformation pseudoelasticity and deformation behavior in a Ti-50.6at\% Ni alloy. Scr. Metall. 15, 287-292 (1981)

4. H. Sehitoglu, J. Jun, X. Zhang, I. Karaman, Y. Chumlyakov, H.J. Maier, K. Gall, Shape memory and pseudoelastic behavior of $51.5 \% \mathrm{Ni}-\mathrm{Ti}$ single crystals in solutionized and overaged state. Acta Mater. 49, 3609-3620 (2001)

5. K. Otsuka, X. Ren, Physical metallurgy of Ti-Ni-based shape memory alloys. Prog. Mater Sci. 50, 511-678 (2005)

6. Y. Liu, Z.L. Xie, J. Van Humbeeck, L. Delaey, Effect of texture orientation on the martensite deformation of NiTi shape memory alloy sheet. Acta Mater. 47, 645 (1999)

7. Y. Liu, Z.L. Xie, Twinning and detwinning of $\langle 011\rangle$ type II twin in shape memory alloy. Acta Mater. 51(18), 5529-5543 (2003)

8. Z.L. Xie, Y. Liu, HRTEM study of $\langle 011\rangle$ type II twin in NiTi shape memory alloy. Philos Mag. 84(32), 3497-3507 (2004)

9. T. Ezaz, H. Sehitoglu, H.J. Maier, Energetics of twinning in martensitic NiTi. Acta Mater. 59, 5893 (2011)

10. M. Nishida, H. Ohgi, I. Itai, A. Chiba, K. Yamauchi, Electron microscopy studies of twin morphologies in B19' martensite in the Ti-Ni shape memory alloy. Acta Metall. Mater. 43, 1219-1227 (1995)

11. T. Ezaz, H. Sehitoglu, Type II detwinning in NiTi. Appl. Phys. Lett. 98, 141906 (2011)

12. T. Ezaz, H. Sehitoglu, W. Abuzaid, H.J. Maier, Higher order twin modes in martensitic NiTi-the $(20 \overline{1})$ case. Mater. Sci Eng. A 558, 422 (2012)

13. T. Onda, Y. Bando, T. Ohba, K. Otsuka, Electron microscopy study of twins in martensite in a Ti-50.0 at $\% \mathrm{Ni}$ alloy. Mater. Trans JIM 33, 354 (1992)

14. J.X. Zhang, M. Sato, A. Ishida, Deformation mechanism of martensite in Ti-rich Ti-Ni shape memory alloy thin films. Acta Mater. 54, 1185 (2006) 
15. R.F. Hamilton, S. Dilibal, H. Sehitoglu, H.J. Maier, Underlying mechanism of dual hysteresis in NiMnGa single crystals. Mater.Sci. Eng. A 528, 1877-2281 (2011)

16. S. Dilibal, H. Sehitoglu, R.F. Hamilton, H.J. Maier, Y. Chumlyakov, On the volume change in Co-Ni-Al during pseudoelasticity. Mater. Sci. Eng. A 528, 2875-2881 (2011)

17. J. Carroll, L.N. Brewer, C.C. Battaile, B.L. Boyce, J.M. Emery, The effect of grain size on local deformation near a void-like stress concentration. Int. J. Plast. 39, 46-60 (2012)

18. J. Carroll, W.Z. Abuzaid, J. Lambros, H. Sehitoglu, On the interactions between strain accumulation, microstructure, and fatigue crack behavior. Int. J. Fract. 180(2), 223-241 (2013)

19. C. Efstathiou, H. Sehitoglu, J. Carroll, J. Lambros, H.J. Maier, Full-field strain evolution during intermartensitic transformations in single crystal NiFeGa. Acta Mater. 56(15), 3791-3799 (2008)

20. K. Kim, S. Daly, Martensite strain memory in the shape memory alloy nickel-titanium under mechanical cycling. Exp. Mech. 51, 641-652 (2011)

21. S. Daly, D. Rittel, K. Bhattacharya, G. Ravichandran, Large deformation of Nitinol under shear dominant loading. Exp. Mech. 49, 225-233 (2009)

22. W.H. Peters, W.F. Ranson, Digital imaging techniques in experimental stress analysis. Opt. Eng. 21, 427-432 (1982)

23. C. Efstathiou, H. Sehitoglu, J. Lambros, Multiscale strain measurements of plastically deforming polycrystalline Ti: role of deformation heterogeneities, Int. J. Plast. 26, 93 (2010) 